\title{
Introducción: Hacia una filosofía de la ciudad
}

\section{Introduction: Towards a Philosophy of the City}

PAULA C. PEREIRA (Universidade do Porto), DOMINGO GARCÍA-MARZÁ (Universitat Jaume I) y JOSÉ L. LÓPEZ-GONZÁLEZ (Universitat Jaume I)

Pereira, Paula C., García-Marzá, Domingo y López-González, José L. (2020). Introducción: Hacia una filosofía de la ciudad. Recerca. Revista de Pensament i Análisi, $25(2)$, pp. 1-7.

Teniendo en cuenta que la filosofía es hija de la polis, a primera vista, el tema de la filosofía de la ciudad puede no revelar nada nuevo. De hecho, la filosofía y la ciudad cuentan una historia paralela. Es en el ágora, un espacio privilegiado para el ejercicio de la ciudadanía, donde la filosofía se desarrolla como debate de argumentos (Pereira, 2011).

Pero, aunque a lo largo de la historia las ciudades puedan reflejar un deseo civilizatorio, lo cierto es que la ciudad contemporánea refleja muy poco de la realidad política de la polis, de la indiferenciación medieval entre lo público y lo privado o de la racionalidad normativa de la ciudad moderna. Las ciudades en el siglo XX, y especialmente en el siglo XXI, tienen que ver más con las relaciones sociales y económicas de la ciudad industrial y posindustrial. La progresiva mecanización del siglo XIX y su extensión, en el siglo XX, a la automatización y a la digitalización, cambian definitivamente la forma en que nos relacionamos, pensamos y trabajamos —en resumen, cómo vivimos-. Con el establecimiento de un capitalismo de orden económico que afecta a todas las dimensiones de la vida de la ciudad, esta queda delimitada por el poder, revela las contradicciones de las sociedades urbanas diseñadas por los mercados y da lugar, según David Harvey (2003, 2008), a la apropiación privada de la vida y los espacios.

El vertiginoso ritmo de los cambios tecnológicos y climáticos de los últimos cuatro decenios, así como su influencia en los más diversos campos de pensamiento y acción, hacen cada vez más compleja la identificación de las cadenas causales en las nuevas dinámicas y fenómenos urbanos. El refuerzo mutuo y la interacción de las variables globales y locales, la inscripción de hegemonías en los modos de vida y la dilución de las dicotomías sujeto/objeto y 
real/virtual desafían las posibilidades de producción/creación de espacios y experiencias urbanas. Desde este punto de vista, es urgente, en un siglo que es claramente urbano, problematizar una vida cotidiana urbana moldeable por la aceleración social, la tecnología, los principios capitalistas, la regulación biopolítica, la transfiguración de los espacios públicos físicos y la creciente evidencia de la interdependencia ecológica.

Precisamente en este sentido, en los últimos años hemos visto un aumento en el interés por el estudio de los problemas sociales, económicos y políticos que pueden formar parte del campo de la filosofía de la ciudad (Pereira, 2018). Algunos ejemplos son los movimientos de la Primavera Árabe, el 15-M en España, el Occupy Wall Street en los EE. UU. o el Fora Temer en Brasil; la ciberdemocracia; los dispositivos urbanos que promueven procesos más democratizadores; el cosmopolitismo; los fenómenos migratorios y los desplazamientos; la complejidad de la densidad de población; la participación y la sociedad civil; los movimientos sociales; las cuestiones relativas al derecho a la ciudad; el turismo; los conflictos entre el derecho a la ciudad y el impulso institucional del derecho al turismo (López-González, 2018); la aplicación de neurotecnologías a la política y a diferentes actividades sociales (García-Marzá, 2016), entre las que el neuroturismo puede conllevar especiales repercusiones para la ciudad (López-González, 2019); la gentrificación; el transporte y la vivienda; o la nueva pobreza producida por la ciudad fragmentada y anónima.

En efecto, estos problemas - entre otros- alejan definitivamente a la ciudad contemporánea de la ciudad antigua. Y, en consecuencia, obligan cada vez más a territorializar el pensamiento, es decir, a renovarlo, uniendo el hacer de la filosofía -especialmente la filosofía política- con el hacer ciudad. Implican, por tanto, extender y concretar la democracia, superando así la tradicional reducción de lo político a lo institucional y al Estado (Pereira, 2018, 2019).

En este contexto, que puede ser concebido como posnacional siguiendo a Habermas (2006), efectivamente, los Estados ven desbordada a menudo su capacidad para hacer frente a la resolución de conflictos. Comparten poder con instituciones, entre ellas los global players de carácter no estatal, a las que no siempre pueden alcanzar mediante sus mecanismos de coerción tradicionales. Sin embargo, sobre ellas recae una exigencia de legitimación social. Y, en este sentido, es tarea de la ética, en el marco de la filosofía de la ciudad, poner de manifiesto que a la dimensión normativa que contiene esa exigencia de legitimación ya no puede llegarse mediante la lógica ideal-realidad, sino a través de una reconstrucción del saber intuitivo, de las competencias y de las capacidades que los participantes en las actividades sociales ya disponen para la reso- 
lución de conflictos (García-Marzá, 2017). Desde este punto de vista, ofrecer razones para justificar alternativas a la resolución de conflictos que no se agoten en el aparato administrativo-jurídico constituye una tarea de la que pueden dar cuenta las bases éticas de una democracia de doble vía, en la que Estado y sociedad civil se complementan. Una tarea para la que las exigencias morales, que se concretan en la demanda de transparencia y en la participación, ofrecen un sustento normativo mínimo a partir del cual fundamentar una de las acciones concretas que pueden contribuir a la resolución de los conflictos que se presentan en las ciudades: la creación de espacios de participación con todos los afectados por las actividades institucionales (García-Marzá, 2020).

No podemos olvidar que, en la actualidad, una buena parte de los problemas contemporáneos — de mayor impacto- emergen en los espacios urbanos. De este modo, la territorialización del pensamiento, como profundización de los poderes más colaborativos y participativos, se articula, siguiendo a Lefebvre (1968), con la comprensión de la ciudad como trabajo y valor de uso - y no como valor de cambio-, transformando la práctica industrial en práctica urbana. Una práctica, avanzamos, que requiere el necesario reposicionamiento espacial de la filosofía -otra forma de decir y aclarar la territorialización del pensamiento- para comprender las dimensiones contemporáneas del habitar a través de las relaciones fundamentales entre el ciudadano, la política y el espacio.

La filosofía de la ciudad, tal como la entendemos aquí, nace, por lo tanto, en buena parte, con la ciudad posindustrial, constituyéndose como algo nuevo o renovado para pensar el tiempo que nos fue dado para vivir. Se trata de un compromiso de la filosofía contemporánea con una ciudad que parece prometer desestabilizar áreas conceptuales más tradicionales en las teorizaciones de la ciudad y también confirmar, en una lectura integrada, que la condición urbana sufre, en la misma proporción de su contribución, el impacto inquietante de las transformaciones globales y, a pesar de ello —o por ello mismo-, reúne las condiciones que pueden responder a uno de los mayores desafíos del presente y del futuro: las metrópolis y la urbanización imparable, ahora radicalizada por la pandemia provocada por un nuevo coronavirus.

Cuando se consideró este número monográfico, no podíamos imaginar que una pandemia llegaría, de manera abrumadora, a confirmar y profundizar, en la crudeza de su desnudez, las patologías sociales y muchos de los problemas políticos, éticos, económicos, ecológicos o tecnológicos anunciados y debatidos en las últimas décadas. Siempre es un riesgo hablar o escribir sobre un even- 
to en curso, del que no sabemos claramente cómo comenzó y menos aún sobre su impacto en todas las dimensiones de nuestras vidas. No obstante, esta pandemia reitera la relevancia de la filosofía de la ciudad. La pandemia es esencialmente urbana, su contexto es el de la urbanización mundial que amenaza la justicia espacial y muestra los frágiles fundamentos sociales sobre los que ya vivíamos.

Las ciudades se han vuelto silenciosas, el espacio público se ha reducido y el déficit democrático se ha agudizado. Las ciudades pandémicas y pospandémicas requieren, cada vez más, del compromiso de la filosofía con la ciudad, es decir, un compromiso del pensamiento con el acontecimiento, de los filósofos con las comunidades y los responsables locales, así como de la política con la sociedad y la ciencia para el desarrollo de nuevas ideas y la reconfiguración de los conceptos; porque lo que está en juego, de manera urgente, son imperativos - como la justicia, los derechos humanos, el derecho a la ciudad, la solidaridad, la gobernanza y la libertad - para la construcción de ciudades más justas y sostenibles. Los artículos presentados a continuación reflejan este modo de entender la filosofía de la ciudad.

En la perspectiva presentada por Jorge León Casero y Julia Urabayen en el artículo «Paradigmas de producción urbana y sus resistencias: ciudad, metrópolis y postmetrópolis», las lógicas de producción y gobernanza social son desafiadas por la movilización de puntos de resistencia - o centros nerviososque representan conceptualmente cada paradigma de reproducción del espacio urbano - ciudad, metrópoli y postmetrópoli. En las postmetrópolis occidentales de hoy, los sujetos políticos antagónicos que, con mayor eficacia, pueden superar la crisis del espacio urbano como medio de subjetivación política emergen de las ciudades inteligentes y los procesos de regeneración urbana. Como defienden los autores, en una sociedad cibernética el antagonismo social debe ser reequilibrado no solo desde un trabajo inmaterial, sino también desde un proyecto de apropiación de tecnologías de plataforma. Es aquí donde se configura la relevancia de un sujeto político que establece la eficiencia de su agencia a través del uso de redes de información en lugar de apoderarse de las calles de la ciudad.

En el texto «Experiencia subjetiva del espacio urbano y alienación. Un análisis a partir de la generalización de los dispositivos de navegación digital», Gonzalo Velasco ve el espacio urbano como principal objeto de estudio de la indagación filosófico-antropológica. Este es el espacio que permite la relación - generalmente desvinculada de la teorización filosófica- de la conciencia y la agencia humanas con el entorno concreto de artefactos y objetos que se en- 
cuentra, a mayor escala, en la ciudad. El uso de smartphones como herramienta de orientación a través de sistemas de navegación GPS sirve para modificar la experiencia subjetiva de la ciudad y también sirve como análisis de las posibilidades de apropiación/producción vivencial y estética del espacio urbano. Como nicho tecnológico capaz de potenciar las posibilidades de la vida y la convivencia humanas, el espacio urbano es un mediador entre los ideales de la civilización y la vida cotidiana. Pero también puede ser un condicionante de los viajes, la orientación y la representación simbólica de la vida urbana. La perniciosa superposición del espacio representado - con su multitud de datos y aplicaciones inteligentes - al espacio experimentado genera el peligro de alienar la experiencia inmanente del sujeto comprometiendo tanto su autonomía en la experiencia urbana como sus posibilidades de agencia. Es crucial, por tanto, contemplar formas de salvaguardar la autonomía y una experiencia estética activa —cualidades que el autor invoca en la figura del flâneur de Baudelaire. Y más en estos tiempos urbanos donde el ritmo vertiginoso y la hiperconexión pueden privarnos paradójicamente de la sensorialidad y del ejercicio del derecho a la ciudad.

La filosofía de la ciudad moviliza la relacionalidad constitutiva del estar en el mundo y con el mundo. Su carácter interdisciplinario también contribuye a afirmar esta dinámica relacional. En el artículo «Climate Change and Conservation Biology as it Relates to Urban Environments», Samantha Noll y Michael Goldsby refuerzan esta relación con una reflexión centrada en la ecología urbana. Al proponer un nuevo marco conceptual para entender la conservación de ecosistemas silvestres, concilian los objetivos de conservación y restauración ambiental a través de la reconceptualización de lo que es un ecosistema. En este proceso, tanto la mitigación del impacto de la actividad humana en el clima como la adaptación a los efectos del cambio climático por parte de los ciudadanos, la infraestructura y las ecologías deben perseguirse en un diálogo entre la biología de la conservación, la ecología comunitaria, la filosofía ambiental y la filosofía de la ciudad. Las zonas urbanas, al albergar a más de la mitad de la población mundial, asumen gran parte de la responsabilidad del cambio climático - en una relación directa entre el grado de urbanización y las emisiones de gases de efecto invernadero-. Sin embargo, debido a la posibilidad de racionalizar los medios y aplicar estrategias de sostenibilidad, así como a la voluntad política de los residentes de abordar estas cuestiones, es también de las zonas urbanas de donde se esperan soluciones o mitigación del cambio climático. 
En definitiva, la ciudad se convierte en un espacio democrático, un espacio público, capaz de convertir las capacidades de participación en recursos de poder comunicativo para la resolución de problemas, más aún, para la integración de la responsabilidad social, económica y ecológica tanto de los individuos como de sus instituciones. Esta es la fuerza de la ciudad como puente entre el Estado y la sociedad civil.

\section{BIBLIOGRAFÍA}

García-Marzá, Domingo (2016). Neuroética aplicada: Las consecuencias prácticas del neuropositivismo. Pensamiento, $72(273), 881-900$.

García-Marzá, Domingo (2017). Sociedad civil global. Hacia una ética de las instituciones. Filosofia. Revista da Faculdade de Letras da Universidade do Porto, 34, 31-44.

García-Marzá, Domingo (2020). Deliberative democracy: An upgrade proposal. En Flesher, Cristina y Feenstra, Ramon A. (Eds.). Routledge Handbook of Contemporary European Social Movements (8999). London: Routledge.

Habermas, Jürgen (2006). El Occidente escindido. Madrid: Trotta.

Harvey, David (2003). The New imperialism. Oxford: Oxford University Press.

Harvey, David (2008). The Right to the City. New Left Review, 53, September-October, 23-40.

Lefebvre, Henri (1968). Le droit à la ville. Paris: Anthropos.

López-González, José L. (2018). Del código ético a la convención internacional. Un panorama crítico de la organización mundial del turismo desde la perspectiva del cosmopolitismo. Oxímora. Revista internacional de Ética y Política, 12, 205-223.

López-González, José L. (2019). El neuroturismo en la era de la aceleración social: una aproximación a sus implicaciones éticas. En Cañada, Ernest y Murray, Iván (Eds.). Turistificación global: perspectivas críticas en turismo (145-160). Barcelona: Icaria. 
Pereira, Paula C. (2011). Condição humana e condição urbana. Porto: Afrontamento.

Pereira, Paula C. (2018). Philosophy of the City. En Pereira, Paula C. y Couto, Maria J. (Org.). Philosophy, City and Public Space (151-162). Porto: Afrontamento.

Pereira, Paula C. (2019). City and common space. En Meagher, Sharon, Noll, Samantha y Biehl, Joseph (Eds.) The Routledge Handbook on Philosophy of the City (253-262). London and New York: Routledge. 